\title{
An quantificational method for 51 pesticide residues determination in Pu'er tea by LC-MS/MS
}

\section{WU Xu-dong, CHEN Ze-yu, ZHANG Jian-kang, MAN Hong-ping, CHE Tao, LV Sheng, MAO Jing-chun}

Pu'er Comprehensive Technical Testing Center

Abstract: Pesticide residues in tea is a major issue due to their widely used in tea cultivation. Thus, to protect consumers, an appropriate method for determination of their residues in tea should be done. In this study, a method for the simultaneous determination of 51 pesticides in tea was developed and validated. The tea sample was extracted by acetonitrile and purified SPE clearnet TPT column followed by liquid chromatography coupled to tandem mass spectrometry (LC-MS/MS) with multi-reaction monitoring (MRM) mode. The method was validated according to the linearity, limit of detention, precision, the percentage of recovery at three different spike levels. The linear concentration range used was $10-100 \mathrm{ng} / \mathrm{mL}$, the square of Correlation coefficient $\mathrm{r}^{2}$ was more than $>0.995$. Recoveries were adequate being in the acceptable range of $72-89 \%$ and RSD of $<19 \%$ for all the analytes at three level of $0.01,0.05$ and $0.11 \mathrm{mg} / \mathrm{kg}$, the LOD of all chemicals from $0.001 \mathrm{mg} / \mathrm{kg}$ to $0.01 \mathrm{mg} / \mathrm{kg}$. The method was applied for the determination for 400 tea samples collected from Pu'er which contain green tea and black tea. Among the analyzed samples , $36 \%$ samples had Imidacloprid and 25\% sample contain Acetamiprid, which were at a level below the European Union maximum residue levels (EU-MRLs). The information would be beneficial for Pu'er tea exporters.

Key words: multi-pesticide residue determination; puer tea; LC-MS/MS

\section{Introduction}

Nowadays ,tea has become a popular beverage throughout the world with it has pleasant flavours and antioxidant quality that is good for human health.Thus, Market demand for tea gradually improved. To ensure the high yield of tea ,different pesticides were inevitably used in tea growing.

Pesticides are commonly used in the control of weeds and crop diseases. Especially insecticides, herbicides and plant growth regulators are spread on tea plantations ${ }^{1-2}$. The presence of pesticide residues in tea is becoming a priority concern for the consumer's as its accumulation will have a risk to health ${ }^{3-4}$. In order to protect the consumer's health and also promote the development of tea industry, the content of pesticide residues in tea should be less than the maximum residue level. Thus, establishing a reliable analysis method to monitor and detect of pescitides in tea products 
(http://creativecommons.org/licenses/by-nc/4.0/), which permits unrestricted use, distribution, and reproduction in any medium, provided the original work is properly cited.

is very urgent.

So far Various determination strategies and techniques for pesticides detection in tea have been published, including GC-electron capture

detection (GC-ECD), GC-flame photometric detection (GC-FPD), GC with a flame ionization detector (GC-FID), HPLC-fluorescence detector (HPLC-FLD),GC-MS and LC-MS/MS etc ${ }^{5-16}$. Among the all the methods, Conventional liquid Chromatography high effective separation function coupled with MS a validated method to conformation compound is becoming a new routine pesticide residue determination programs, which exhibits higher sensitivity and specificity ${ }^{17-18}$.

The purpose of this article is concentrate on developing a multi-pesticide residue analytical method for the determination of fifty-one pesticides in tea using LC/MS/MS. Finaly, the established method was used for the residue analysis of 400 tea samples collected from different area in Pu'er.

\section{Reagents and Materials}

Reference standards of 51 Pesticides were obtained from Agro-Environmental Protection Institute Ministry of agriculture (China). chromatography grade methanol, acetonitrile, and formic acid were from J. T. Baker . Solid phase column(SPE,) were purchased from Agela Technologies. Pesticide standard stock solutions were respectively diluted by methanol to a concentration of $10 \mathrm{ug} / \mathrm{mL}$ and stored at a temperature of -20 degrees centigrade until use . A mixed working solution of $100 \mathrm{ng} / \mathrm{mL}$ in methanol containing all selected Pesticide standard was prepared. Intermediate working solutions ,Matrix matched calibration standards were prepared by diluting mixed standard solution to reach the final concentrations of $10,20,40,60,80$, and $100 \mathrm{ng} / \mathrm{mL}$.

\subsection{Samples}

A total of 401 tea samples were purchased from the local market in Pu'er. Among the sample 200 green tea and 200 black tea were collected from Pu'er, one organic tea was obtained from ZUXIANG tea garden(an organic tea garden which has conferred on the organic food certification ).All collected samples were smashed into powder by the use of shredding machine and stored in dryer until to analysis.

\subsection{Sample Extraction and purification}

2 grams of tea powder was weighted and transferred into a 50-mL centrifuge tube. Then $15 \mathrm{~mL}$ acetonitrile was added into the centrifuge tube. The centrifuge tube was shaken vigorously for $30 \mathrm{~min}$ by constant temperature vibrato (IKA,KS4000i,Germany) at $400 \mathrm{rpm}$. Afterward, the mixture was centrifuged for $5 \mathrm{~min}$ at $4000 \mathrm{rpm}$. All supernatant was taken into a $50 \mathrm{~mL}$ specimen bottle .To complete extraction of pescides from the tea, $15 \mathrm{~mL}$ acetonitrile was used to re-extract the residual of sample, and repeat above program, then coupled first supernatant with the later .The supernatant was concentrated by using the Rotary Evaporator(Buch, R-215,Switzerland) with $40{ }^{\circ} \mathrm{C}$ water bath reach to about $1 \mathrm{~mL}$. The concentrated extract was passed through activated solid phase extraction (SPE) column, then the target analyte was eluted from the SPE column by $30 \mathrm{~mL}$ mixed solution of acetonitrile : methylbenzene (toluene)(3:1, v/v) under the atmospheric pressure. The eluant was dryed by using the Rotary Evaporator. $1 \mathrm{~mL}$ of the mobile phase mixture (20\% mobile phase A and 80\% mobile phase B) was used to re-dissolve the extracts and then transferred into $2 \mathrm{~mL}$ vial for analysis at LC-MS/MS after filtration with disposable nylon membrane filter (0.22 micron). The amount of the sample in the final extract was equivalent to $2 \mathrm{~g} / \mathrm{mL}$.

\section{Instrumental Analysis}




\subsection{LC-MS/MS conditions}

a liquid chromatography-mass spectrometry system was used for the identification and quantification of the selected pesticides. The system was consisted of liquid chromatography(Agilent Technologes, 1260 infinity, USA) and a triple quadrupole mass spectrometer (Applyed Biology Corporation, 3200, USA). Separation was performed using a Phenomenex kinetex column $(2.6 \mathrm{u}, \mathrm{C} 18,100 \times 2.1 \mathrm{~mm})$ with column oven temperature was $40{ }^{\circ} \mathrm{C}$. the injection volume was $10 \mu \mathrm{L}$ and the total run time was $27 \mathrm{~min}$. The analysis was carried out in gradient mode, the program was 0-3min, 70\% mobile phase $\mathrm{B}$; 3-9min, $60 \%$ mobile phase $\mathrm{B}$; $9-15 \mathrm{~min}, 40 \%$ mobile phase $\mathrm{B} ; 15-23 \mathrm{~min}, 1 \%$ mobile phase $\mathrm{B} ; 23-27 \mathrm{~min}, 99 \%$ mobile phase $\mathrm{B}$. Mobile phase A is acetonitrile and the mobile phase $\mathrm{B}$ is consisted of water containing $0.1 \%$ formic acid. The mobile phase flow rate was $0.4 \mathrm{~mL} / \mathrm{min}$;

The mass spectrometry system was a triple quadrupole which was equipped with an electro spray ionization (ESI) source operated at the positive ion mode. The operating conditions were as follows: sheath gas (nitrogen) pressure was 30 arbitrary units; Auxiliary gas (nitrogen) pressure was 10 arbitrary units; spray voltage was $5500 \mathrm{~V}$; capillary temperature was $425{ }^{\circ} \mathrm{C}$. The collision gas pressure was 1.5 mTorr. The acquisition was made in the mutiple reaction monitoring (MRM) mode. The retention times and LC-MS/MS parameters for the target pesticides are presented in Table 1.

Table 1 LC-MS/MS parameters and retention times for the target pesticides

\begin{tabular}{cccccccc}
\hline pesticides & $\begin{array}{c}\text { RT } \\
(\mathrm{min})\end{array}$ & $\begin{array}{c}\text { Quantification } \\
\text { ion pair(m/z) }\end{array}$ & $\begin{array}{c}\text { DP } \\
(\mathrm{V})\end{array}$ & $\begin{array}{c}\text { CE } \\
(\mathrm{V})\end{array}$ & $\begin{array}{c}\text { Conformation } \\
\text { ion pair(m/z) }\end{array}$ & $\begin{array}{c}\text { DP } \\
(\mathrm{V})\end{array}$ & $\begin{array}{c}\text { CE } \\
(\mathrm{V})\end{array}$ \\
\hline Imidacloprid & 5.53 & $256.2 / 209.9$ & 41 & 23 & $256.2 / 175.2$ & 41 & 23 \\
Diflubenzuron & 12.81 & $311.0 / 158.2$ & 26 & 19 & $311.0 / 141.2$ & 26 & 47 \\
Carbendazim & 4.35 & $192.2 / 160.2$ & 36 & 27 & $192.2 / 132.1$ & 36 & 41 \\
Methomyl & 4.56 & $163.1 / 88.1$ & 16 & 13 & $163.1 / 106.0$ & 16 & 13 \\
Thiamethoxam & 5.00 & $292.0 / 211.0$ & 51 & 17 & $292.0 / 181.0$ & 51 & 31 \\
Hexythiazox & 18.62 & $353.0 / 228.0$ & 56 & 21 & $353.0 / 168.0$ & 56 & 33 \\
Phoxim & 16.81 & $299.1 / 129.1$ & 36 & 19 & $299.1 / 77.1$ & 36 & 49 \\
Carbofuran & 7.34 & $222.2 / 123.1$ & 26 & 29 & $222.2 / 165.2$ & 26 & 15 \\
Propoxur & 7.23 & $210.1 / 111.0$ & 21 & 19 & $210.1 / 168.1$ & 21 & 11 \\
Tricyclazole & 5.96 & $190.0 / 163.0$ & 46 & 31 & $190.0 / 136.0$ & 46 & 37 \\
Acetamiprid & 5.75 & $223.2 / 126.1$ & 36 & 29 & $223.2 / 99.1$ & 36 & 47 \\
Flufenoxuron & 19.04 & $489.1 / 158.2$ & 61 & 25 & $489.1 / 141.2$ & 61 & 65 \\
Propiconazole & 13.77 & $342.1 / 159.1$ & 46 & 43 & $342.1 / 69.1$ & 46 & 33 \\
Tebuconazole & 12.47 & $308.0 / 70.0$ & 21 & 39 & $308.0 / 125.0$ & 21 & 47 \\
Thiabendazole & 4.68 & $202.1 / 175.1$ & 56 & 35 & $202.1 / 131.2$ & 56 & 43 \\
Cartap hydrochloride & 0.65 & $150.0 / 105.0$ & 33 & 22 & $150.0 / 61.0$ & 31 & 34 \\
Imidaclothiz & 5.64 & $262.1 / 181.1$ & 30 & 21 & $262.1 / 122.1$ & 29 & 50 \\
Trichlorfon & 5.10 & $257.1 / 221.1$ & 34 & 18 & $257.1 / 109.2$ & 32 & 27 \\
Aldicarb & 6.18 & 208.2116 .1 & 11 & 11 & $208.2 / 89.1$ & 11 & 21 \\
Atrazine & 7.87 & $216.1 / 174.1$ & 51 & 19 & $216.1 / 104.0$ & 51 & 39 \\
Benalaxyl & 14.58 & $326.2 / 148.2$ & 31 & 29 & $326.2 / 91.1$ & 31 & 49 \\
Difenoconazole & 15.46 & $406.2 / 251.1$ & 56 & 37 & $406.2 / 253.1$ & 56 & 33 \\
Diniconazole & 13.47 & $326.0 / 70.0$ & 51 & 45 & $326.0 / 159.0$ & 51 & 39 \\
\hline & & & & & & &
\end{tabular}




\begin{tabular}{cccccccc}
\hline Epoxiconazole & 11.84 & $330.0 / 121.0$ & 36 & 27 & $330.0 / 101.0$ & 36 & 63 \\
Fenazaquin & 18.62 & $307.0 / 161.0$ & 41 & 31 & $307.0 / 147.0$ & 41 & 35 \\
Fenobucarb & 9.70 & $208.0 / 152.0$ & 6 & 13 & $208.0 / 95.0$ & 6 & 21 \\
Hexaconazole & 12.88 & $314.0 / 70.0$ & 36 & 39 & $314.0 / 159.0$ & 26 & 37 \\
Isoprocarb & 8.40 & $194.2 / 95.1$ & 31 & 20 & $194.2 / 137.2$ & 31 & 13 \\
Isoproturon & 8.20 & $207.2 / 72.1$ & 46 & 29 & $207.2 / 46.1$ & 46 & 31 \\
Methamidophos & 0.99 & $142.0 / 94.0$ & 31 & 19 & $142.0 / 125.0$ & 31 & 17 \\
Metolcarb & 6.60 & $166.2 / 109.1$ & 16 & 15 & $166.2 / 94.2$ & 16 & 37 \\
Monocrotophos & 4.68 & $224.0 / 127.0$ & 46 & 21 & $224.0 / 98.0$ & 46 & 17 \\
Methidathion & 9.80 & $303.0 / 145.1$ & 21 & 15 & $303.0 / 85.2$ & 21 & 29 \\
Myclobutanil & 11.30 & $289.0 / 70.0$ & 31 & 33 & $289.0 / 125.0$ & 31 & 41 \\
Penconazole & 12.90 & $284.0 / 159.0$ & 41 & 39 & $284.0 / 70.0$ & 41 & 29 \\
Piperonyl Butoxide & 17.67 & $356.2 / 177.2$ & 21 & 28 & $356.2 / 119.0$ & 21 & 35 \\
Pirimicarb & 5.21 & $239.2 / 72.1$ & 33 & 34 & $239.2 / 182.2$ & 33 & 21 \\
Prochloraz & 10.52 & $376.1 / 308.0$ & 21 & 17 & $376.1 / 70.1$ & 21 & 37 \\
Propargite & 19.26 & $368.0 / 231.0$ & 6 & 17 & $368.0 / 175.0$ & 6 & 21 \\
Propiconazole & 13.77 & $342.1 / 159.1$ & 46 & 43 & $342.1 / 69.1$ & 46 & 33 \\
Triadimefon & 11.59 & $294.0 / 197.0$ & 36 & 21 & $294.0 / 225.0$ & 36 & 19 \\
Triadimenol & 9.69 & $296.1 / 70.1$ & 11 & 19 & $296.1 / 227.2$ & 11 & 15 \\
Pyriproxyfen & 18.20 & $322.0 / 96.0$ & 26 & 21 & $322.0 / 185.0$ & 26 & 29 \\
Quinoxyfen & 16.40 & $308.0 / 197.0$ & 21 & 43 & $308.0 / 162.0$ & 21 & 57 \\
Rotenone & 13.95 & $395.0 / 213.0$ & 66 & 29 & $395.0 / 192.0$ & 66 & 31 \\
Simazine & 6.60 & $202.1 / 132.1$ & 46 & 25 & $202.1 / 124.3$ & 46 & 25 \\
Pymetrozine & 3.12 & $218.0 / 105.0$ & 51 & 27 & $218.0 / 78.0$ & 51 & 47 \\
Propanil & 9.26 & $218.0 / 126.9$ & 52 & 55 & $218.0 / 161.7$ & 57 & 35 \\
Furathiocarb & 17.99 & $383.2 / 195.4$ & 17 & 36 & $383.2 / 167.1$ & 26 & 32 \\
Chlorotoluron & 7.66 & $213.0 / 72.3$ & 55 & 27 & $213.0 / 140.1$ & 38 & 34 \\
Fenchlorphos & 22.24 & $321.0 / 125.1$ & 34 & 29 & $321.0 / 288.7$ & 32 & 27 \\
Prometryn & 7.65 & $242.0 / 158.2$ & 67 & 28 & $242.0 / 200.1$ & 54 & 27 \\
\hline & & & & & & &
\end{tabular}

\subsection{Method Validation}

To ensure analysis credibility. The analytical method was validated by evaluating the linearity, accuracy, precision and limit of detection (LOD).

\subsection{Accuracy and Precision}

The method accuracy and precision was determined by recovery tests which using organic tea samples spiked with mixed standard solution at $0.01,0.05$ and $0.1 \mathrm{mg} / \mathrm{kg}$. Recovery test was done by analyzing three replicates . Organic tea was spiked prior to the extraction procedure by the addition of a mixed pesticide standard working solution to reach the final fortification levels. After fortification, the sample was equilibrated by settling for $30 \mathrm{~min}$ before to extraction in order to ensure the sufficient contact of the analytes with the organic tea. Then, the samples were prepared and analysed according to the method described earlier.

Precision in case of repeatability (RSD) was determined at fortification levels of $0.01,0.05$, and $0.10 \mathrm{mg} / \mathrm{kg}$ with three 
replicates. The Mean recovery rate and RSD were listed in the table 2.

Table 2 Mean recovery (\%) and RSD (\%) of the selected pesticides in organic tea at different fortification levels $(\mathrm{n}=3)$

Name of pesticide

\begin{tabular}{lllll}
\multicolumn{4}{c}{ Fortification } \\
\hline $0.01 \mathrm{mg} / \mathrm{kg}$ & \multicolumn{2}{c}{$0.05 \mathrm{mg} / \mathrm{kg}$} & $0.1 \mathrm{mg} / \mathrm{kg}$ \\
\hline Mean RSD & Mean & RSD & Mean & RSD
\end{tabular}

$(\%) \quad(\%) \quad(\%) \quad(\%) \quad(\%) \quad(\%)$

\begin{tabular}{|c|c|c|c|c|c|c|}
\hline Imidacloprid & 81 & 12 & 87 & 6 & 84 & 5 \\
\hline Diflubenzuron & 80 & 7 & 83 & 11 & 79 & 9 \\
\hline Carbendazim & 76 & 10 & 77 & 12 & 88 & 7 \\
\hline Methomyl & 88 & 19 & 81 & 13 & 89 & 8 \\
\hline Thiamethoxam & 79 & 18 & 84 & 9 & 83 & 14 \\
\hline Hexythiazox & 74 & 9 & 82 & 18 & 77 & 11 \\
\hline Phoxim & 80 & 14 & 78 & 9 & 85 & 7 \\
\hline Carbofuran & 83 & 6 & 76 & 8 & 80 & 4 \\
\hline Propoxur & 86 & 15 & 77 & 8 & 79 & 12 \\
\hline Tricyclazole & 82 & 6 & 86 & 11 & 81 & 5 \\
\hline Acetamiprid & 83 & 8 & 77 & 4 & 78 & 13 \\
\hline Flufenoxuron & 84 & 7 & 80 & 4 & 84 & 12 \\
\hline Propiconazole & 83 & 8 & 82 & 4 & 75 & 14 \\
\hline Tebuconazole & 83 & 8 & 86 & 3 & 78 & 6 \\
\hline Thiabendazole & 87 & 9 & 83 & 7 & 81 & 11 \\
\hline Cartap hydrochloride & 86 & 7 & 79 & 8 & 78 & 12 \\
\hline Imidaclothiz & 80 & 8 & 71 & 6 & 84 & 6 \\
\hline Trichlorfon & 74 & 15 & 81 & 16 & 77 & 9 \\
\hline Aldicarb & 83 & 6 & 87 & 8 & 74 & 2 \\
\hline Atrazine & 73 & 7 & 75 & 5 & 87 & 4 \\
\hline Benalaxyl & 76 & 5 & 80 & 6 & 78 & 4 \\
\hline Difenoconazole & 80 & 8 & 87 & 8 & 86 & 13 \\
\hline Diniconazole & 82 & 9 & 77 & 5 & 73 & 7 \\
\hline Epoxiconazole & 79 & 6 & 83 & 7 & 81 & 7 \\
\hline Fenazaquin & 80 & 4 & 87 & 8 & 76 & 6 \\
\hline Fenobucarb & 79 & 6 & 87 & 14 & 89 & 6 \\
\hline Hexaconazole & 89 & 9 & 84 & 7 & 79 & 6 \\
\hline Isoprocarb & 83 & 7 & 78 & 12 & 80 & 9 \\
\hline Isoproturon & 83 & 11 & 87 & 7 & 81 & 6 \\
\hline Methamidophos & 85 & 12 & 72 & 6 & 75 & 5 \\
\hline Metolcarb & 79 & 11 & 77 & 7 & 75 & 10 \\
\hline Monocrotophos & 84 & 2 & 80 & 12 & 88 & 7 \\
\hline Methidathion & 74 & 11 & 81 & 9 & 83 & 3 \\
\hline Myclobutanil & 86 & 6 & 78 & 11 & 89 & 10 \\
\hline Penconazole & 85 & 5 & 80 & 19 & 79 & 8 \\
\hline
\end{tabular}




\begin{tabular}{ccccccc} 
Piperonyl Butoxide & 80 & 12 & 76 & 4 & 81 & 5 \\
Pirimicarb & 83 & 5 & 85 & 5 & 81 & 6 \\
Prochloraz & 81 & 5 & 87 & 5 & 83 & 3 \\
Propargite & 89 & 9 & 81 & 8 & 80 & 7 \\
Propiconazole & 85 & 6 & 79 & 7 & 81 & 3 \\
Triadimefon & 81 & 9 & 87 & 12 & 79 & 4 \\
Triadimenol & 85 & 16 & 88 & 5 & 87 & 5 \\
Pyriproxyfen & 82 & 8 & 75 & 17 & 78 & 12 \\
Quinoxyfen & 80 & 13 & 77 & 9 & 83 & 12 \\
Rotenone & 81 & 11 & 83 & 6 & 87 & 8 \\
Simazine & 86 & 12 & 81 & 10 & 81 & 9 \\
Pymetrozine & 81 & 13 & 78 & 5 & 76 & 4 \\
Propanil & 79 & 11 & 88 & 13 & 84 & 10 \\
Furathiocarb & 74 & 18 & 87 & 6 & 89 & 5 \\
Chlorotoluron & 85 & 9 & 83 & 6 & 88 & 9 \\
Fenchlorphos & 80 & 12 & 77 & 16 & 78 & 8 \\
Prometryn & 88 & 17 & 89 & 13 & 81 & 8 \\
\hline
\end{tabular}

A satisfied accuracy and precision was found for all the analytes . The recovery percentage of $82 \pm 3.98 \%, 81 \pm 4.53 \%$ and $81 \pm 4.38 \%$ for fortification levels of $0.01,0.05$ and $0.1 \mathrm{mg} / \mathrm{kg}$ organic tea sample, respectively. The date of RSD $\leq 19 \%$ for all the chemicals.

\subsection{Calibration Curve and Linearity}

Consideration the factor of matrix effect ${ }^{19}$,calibration curves were prepared by use of matrix-matched standards analyzed. The organic tea was treated to be blank matrix. Linearity was determined by plotting peak areas versus different known concentrations $(10,20,40,60,80$ and $100 \mathrm{ng} / \mathrm{mL})$ and so obtained linear equation and value of regression coefficient $\left(\mathrm{R}^{2}\right)$.

The limit of detection (LOD, $\mathrm{ng} / \mathrm{mL}$ ) was estimated for a response of 3 times the signal-to-noise at the lowest concentration. The calibration parameters and the limit of detection for all the selected pesticides are summarized in Table 3

Table 3 Limit of detection (LOD) and calibration parameters of the selected pesticides for tea

\begin{tabular}{cccccc}
\hline analyte & $\begin{array}{c}\text { LOD } \\
(\mathrm{mg} / \mathrm{kg})\end{array}$ & $\begin{array}{c}\text { Calibration } \\
\text { range }(\mathrm{mg} / \mathrm{kg})\end{array}$ & \multicolumn{3}{c}{ Calibration parameters } \\
\cline { 4 - 6 } & & & slope & intercept & $\mathrm{R}^{2}$ \\
\hline Imidacloprid & 0.001 & $0.01-0.1$ & 43.67 & 125.92 & 0.995 \\
Diflubenzuron & 0.005 & $0.01-0.1$ & 394.22 & 385.34 & 0.998 \\
Carbendazim & 0.001 & $0.01-0.1$ & 1309.81 & 13726.57 & 0.996 \\
Methomyl & 0.001 & $0.01-0.1$ & 1121.56 & 16269.31 & 0.996 \\
Thiamethoxam & 0.001 & $0.01-0.1$ & 348.73 & -4154.60 & 0.995 \\
Hexythiazox & 0.001 & $0.01-0.1$ & 36.73 & -131.43 & 0.997 \\
Phoxim & 0.003 & $0.01-0.1$ & 31.22 & -61.00 & 0.998 \\
\hline
\end{tabular}




\begin{tabular}{|c|c|c|c|c|c|}
\hline Carbofuran & 0.001 & $0.01-0.1$ & 1043.35 & 22359.14 & 0.997 \\
\hline Propoxur & 0.001 & $0.01-0.1$ & 1224.50 & -5575.00 & 0.997 \\
\hline Tricyclazole & 0.001 & $0.01-0.1$ & 1610.36 & 11464.43 & 0.996 \\
\hline Acetamiprid & 0.001 & $0.01-0.1$ & 718.79 & -7423.29 & 0.995 \\
\hline Flufenoxuron & 0.001 & $0.01-0.1$ & 305.01 & 1453.23 & 0.999 \\
\hline Propiconazole & 0.001 & $0.01-0.1$ & 707.02 & 1160.97 & 0.998 \\
\hline Tebuconazole & 0.001 & $0.01-0.1$ & 3944.20 & 17443.29 & 0.995 \\
\hline Thiabendazole & 0.001 & $0.01-0.1$ & 1129.20 & 11593.29 & 0.998 \\
\hline Cartap hydrochloride & 0.001 & $0.01-0.1$ & 26.80 & 596.00 & 0.995 \\
\hline Imidaclothiz & 0.001 & $0.01-0.1$ & 147.76 & 1047.98 & 0.995 \\
\hline Trichlorfon & 0.001 & $0.01-0.1$ & 13.44 & 1145.31 & 0.998 \\
\hline Aldicarb & 0.001 & $0.01-0.1$ & 30.03 & 190.50 & 0.997 \\
\hline Atrazine & 0.001 & $0.01-0.1$ & 1115.79 & 7056.70 & 0.996 \\
\hline Benalaxyl & 0.001 & $0.01-0.1$ & 3455.30 & -1762.80 & 0.997 \\
\hline Difenoconazole & 0.001 & $0.01-0.1$ & 11446.34 & 70853.65 & 0.995 \\
\hline Diniconazole & 0.01 & $0.01-0.1$ & 1999.69 & 512.80 & 0.995 \\
\hline Epoxiconazole & 0.001 & $0.01-0.1$ & 1858.04 & 1721.95 & 0.997 \\
\hline Fenazaquin & 0.001 & $0.01-0.1$ & 2960.42 & 13857.92 & 0.996 \\
\hline Fenobucarb & 0.007 & $0.01-0.1$ & 412.17 & 814.57 & 0.995 \\
\hline Hexaconazole & 0.001 & $0.01-0.1$ & 2590.12 & 754.87 & 0.996 \\
\hline Isoprocarb & 0.001 & $0.01-0.1$ & 601.74 & 3925.76 & 0.999 \\
\hline Isoproturon & 0.001 & $0.01-0.1$ & 8693.72 & 9528.81 & 0.998 \\
\hline Methamidophos & 0.005 & $0.01-0.1$ & 356.89 & 472.53 & 0.997 \\
\hline Metolcarb & 0.001 & $0.01-0.1$ & 753.24 & 4149.61 & 0.997 \\
\hline Monocrotophos & 0.001 & $0.01-0.1$ & 5414.28 & -52628.57 & 0.996 \\
\hline Methidathion & 0.001 & $0.01-0.1$ & 3303.41 & 24236.58 & 0.996 \\
\hline Myclobutanil & 0.001 & $0.01-0.1$ & 2362.62 & -2870.12 & 0.998 \\
\hline Penconazole & 0.001 & $0.01-0.1$ & 759.40 & -1768.90 & 0.998 \\
\hline Piperonyl Butoxide & 0.001 & $0.01-0.1$ & 2504.14 & 7365.85 & 0.998 \\
\hline Pirimicarb & 0.001 & $0.01-0.1$ & 6848.78 & 126951.21 & 0.998 \\
\hline Prochloraz & 0.001 & $0.01-0.1$ & 379.56 & 1040.99 & 0.996 \\
\hline Propargite & 0.001 & $0.01-0.1$ & 580.19 & -1012.19 & 0.997 \\
\hline Propiconazole & 0.001 & $0.01-0.1$ & 707.02 & 1160.97 & 0.998 \\
\hline Triadimefon & 0.001 & $0.01-0.1$ & 410.90 & -116.15 & 0.997 \\
\hline Triadimenol & 0.001 & $0.01-0.1$ & 517.41 & -1433.41 & 0.995 \\
\hline Pyriproxyfen & 0.001 & $0.01-0.1$ & 10416.79 & 9786.25 & 0.997 \\
\hline Quinoxyfen & 0.001 & $0.01-0.1$ & 862.35 & -540.85 & 0.996 \\
\hline Rotenone & 0.002 & $0.01-0.1$ & 487.72 & 661.67 & 0.999 \\
\hline Simazine & 0.001 & $0.01-0.1$ & 1214.26 & -2979.26 & 0.998 \\
\hline Pymetrozine & 0.001 & $0.01-0.1$ & 469.58 & -2014.34 & 0.996 \\
\hline Propanil & 0.001 & $0.01-0.1$ & 22.01 & 381.00 & 0.997 \\
\hline
\end{tabular}




\begin{tabular}{cccccc}
\hline Furathiocarb & 0.003 & $0.01-0.1$ & 787.79 & 12996.61 & 0.996 \\
Chlorotoluron & 0.001 & $0.01-0.1$ & 915.25 & 22354.23 & 0.996 \\
Fenchlorphos & 0.001 & $0.01-0.1$ & 837.12 & 8722.05 & 0.995 \\
Prometryn & 0.001 & $0.01-0.1$ & 2165.64 & -24.93 & 0.996 \\
\hline
\end{tabular}

The limit of detection a found to be from 0.001 to $0.01 \mathrm{mg} / \mathrm{kg}$. Linearity was very good and coefficients of determination were better than 0.995 for all the selected pesticides with matrix-matched calibration standards.

\section{Application of the Method for Real Sample}

The established method was used for the residue analysis of 400 tea samples collected from different area in puer which contain 200 green tea and 200 black tea. The concentrations of pesticide residual in samples were calculated with the help of matrix-matched calibration curve. The results showed that Imidacloprid(36 \% of the total no. of samples) and Acetamiprid ( $25 \%$ of the total no. of samples)frequently appeared in the tea samples, The concentration ranges of residues were $0.005-0.05 \mathrm{mg} / \mathrm{kg}$ (acetamiprid), $0.01-0.05 \mathrm{mg} / \mathrm{kg}$ (imidacloprid). The contents were unexceed the EU-MRLs value: imidacloprid $(0.05 \mathrm{mg} / \mathrm{kg})$ and acetamiprid $(0.1 \mathrm{mg} / \mathrm{kg})$

\section{Conclusions}

In this study, a LC-MS/MS multi-residue method for the analysis of 51 pesticide recidure was developed. Satisfactory accuracy (average recoveries ranged from 72 to $89 \%$ ) and precision ( $\operatorname{RSDr} \leq 19 \%$ ) was found for all the analytes. For the 51 analytes linear calibrations with coefficients $R^{2} \geq 0.995$ were obtained. The limits of detection of the total pesticedes were $<0.005 \mathrm{mg} / \mathrm{kg}$. Thus, the proposed method can be used successfully to monitor multiple pesticide residues in tea. Finally, the analytical method was applied successfully for pesticide residue analysis of 400 tea samples collected from different region in puer. The results revealed that puer tea carry few kinds of pesticide, if the contents were unexceed the maximum residue limit. The information could be useful for policy makers, tea exporters and the public at large.

\section{References}

1. S Seenivasan,N Muraleedharan.Survey on the pesticide residues in tea in south India.Environmental Monitoring \& Assessment,2011,176(1-4):365-371.

2. H Chen, Z Hao, Q Wang. Occurrence and risk assessment of organophosphorus pesticide residues in Chinese tea.Human\&Ecological Risk Assessment,2016, 22(1):28-38.

3. Cynthia K. Colapinto,Tye E. Arbuckle,Lise Dubois. Tea consumption in pregnancy as a predictor of pesticide exposure and adverse birth outcomes:The MIREC Study.Environmental Research 2015,142:77-83.

4. H Chen ,M Pan ,X Liu. Evaluation of transfer rates of multiple pesticides from green tea into infusion using water as pressurized liquid extraction solvent and ultra-performance. Food Chemistry, 2017, $216: 1$.

5. A.Niaz,R.A. Sial1, M. Yaseen, G. A. Mand. Determination of Imidacloprid residues in rice from various districts of Punjab using high performance liquid chromatography. The Journal of Animal \& Plant Sciences, 2016,26(1):170-176.

6. Lou Zhenyun, Chen Zongmao, Luo Fengjian . Determination of 92 pesticide residues in tea by gas chromatography with solid-phase extraction. Chinese journal of chromatography, 2008,26(5):568-576.

7. Lu Xiaolei, Zhou Weilong . Overview of Chinese determination standards of pesticides residues in tea. Journal of 
Food Safety and Quality, 2015, 6(5):1548-1553.

8. Hongping Chen, Guanwei Gao, Pingxiang Liu.Determination of 16 Polycyclic Aromatic Hydrocarbons in Tea by Simultaneous Dispersive Solid-Phase Extraction and Liquid-Liquid Extraction Coupled with gas Chromatography-Tandem Mass Spectrometry. Food analytical methods, 2016, 9: 2374-2384.

9. Chia Chang $\mathrm{Wu}$, Chun $\mathrm{Chu}$, Yei Shung Wang . Multi residue method for high-performance liquid chromatography determination of carbamate pesticides residues in tea samples. Journal of Environmental Science and Health Part B (2009) 44, 58-68.2374-2384.

10. Xin Li,Zhaowei Zhang, Peiwu Li. Determination for major chemical contaminants in tea (Camellia sinensis) matrices. Food Research International. 2013, 53: 649-658.

11. Cai, L. S, Xing, J, Dong, L. Application of polyphenylmethylsiloxane coated fiber for solid-phase microextraction combined with microwave-assisted extraction for the determination of organochlorine pesticides in Chinese teas.Journal of Chromatography. A, 1015(1-2), 11-21.

12. Chen G, Cao P, Liu R. A multi-residue method for fast determination of pesticides in tea by ultra performance liquid chromatography-electrospray tandem mass spectrometry combined with modified QuEChERS sample preparation procedure.Food Chemistry, 125(4), 1406-1411.

13. XX Zeng,YQ Wan,MY Xie. Simultaneous Determination of Organochlorine and Pyrethroid Pesticide Residues in Tea of Jiangxi Province by GC-ECD. Journal of Analytical Science, 2008, 24(6):636-640.

14. Z Lou, Z Chen,F Luo.Determination of 92 pesticide residues in tea by gas chromatography with solid-phase extraction.Chinese Journal of Chromatography,2008,26(5):568-576

15. B Li,F Zeng, Q Dong. Rapid Determination Method for 12 Pyrethroid Pesticide Residues in Tea by Stir Bar Sorptive Extraction-Thermal Desorption-Gas Chromatography. Physics Procedia , 2012 , 25 (22):1776-1780

16. Cho,S.K,Abd El-Aty,A.M,Choi.Effectiveness of pressurized liquid extraction and solvent extraction for the simultaneous quantification of 14 pesticide residues in green tea using GC.Journal of Separation Science,31(10),1750-1760.

17. Zhiqiang Huang, Ying Zhang, Libing Wang. Simultaneous determination of 103 pesticide residues in tea samples by LC-MS/MS. J. Sep. Sci. 2009, 32, 1294- 1301.

18. S Liu, Z Zheng ,F Wei.Simultaneous Determination of Seven Neonicotinoid Pesticide Residues in Food by Ultraperformance Liquid Chromatography Tandem Mass Spectrometry. Journal of Agricultural \& Food Chemistry, 2017, 58 (6):3271-3278.

19. M.D.H.Prodhan,Emmanouil-N,Papadakis,Euphemia Papadopoulou-Mourkidou. Analysis of Pesticide Residues and Their Variability in Cabbage Using QuEChERS Extraction in Combination with LC-MS/MS.Food Anal. Methods.2016.5. 